\title{
Improving the production of AHL lactonase AiiO-AlO6 from Ochrobactrum sp. M231 in intracellular protease-deficient Bacillus subtilis
}

\author{
Rui Xia', Yalin Yang ${ }^{2}$, Xingliang Pan², Chenchen Gao', Yuanyuan Yao', Xuewei Liu², Tsegay Teame', \\ Fengli Zhang ${ }^{1}$, Juan Hu' ${ }^{1}$, Chao Ran², Zhen Zhang ${ }^{2}$, Jihong Liu-Clarke ${ }^{3}$ and Zhigang Zhou ${ }^{\text {** }}$
}

\begin{abstract}
Quorum quenching (QQ) blocks bacterial cell-to-cell communication (i.e., quorum sensing), and is a promising antipathogenic strategy to control bacterial infection via inhibition of virulence factor expression and biofilm formation. QQ enzyme AiiO-AlO6 from Ochrobactrum sp. M231 has several excellent properties and shows biotherapeutic potential against important bacterial pathogens of aquatic species. AiiO-AlO6 can be secretory expressed in Bacillus subtilis via a non-classical secretion pathway. To improve AiiO-AlO6 production, four intracellular protease-deletion mutants of $B$. subtilis $1 \mathrm{~A} 751$ were constructed by individually knocking out the intracellular protease-encoding genes (tepA, ymfH, yrrN and ywpE). The AiiO-AlO6 expression plasmid pWB-AlO6BS was transformed into the B. subtilis 1A751 and its four intracellular protease-deletion derivatives. Results showed that all recombinant intracellular proteasedeletion derivatives (BS $\triangle t e p A, B S \triangle y m f H, B S \triangle y r r N$ and BS $\triangle y w p E$ ) had a positive impact on AiiO-AlO6 production. The highest amount of AiiO-AIO6 extracellular production of BS $\triangle y w p E$ in shake flask reached $1416.47 \mathrm{U} / \mathrm{mL} / \mathrm{OD}_{600}$, which was about $121 \%$ higher than that of the wild-type strain. Furthermore, LC-MS/MS analysis of the degrading products of 3-oxo-C8-HSL by purification of AiiO-AIO6 indicated that AiiO-AlO6 was an AHL-lactonase which hydrolyzes the lactone ring of AHLs. Phylogenetic analysis showed that AiiO-AIO6 was classified as a member of the $\alpha / \beta$ hydrolase family with a conserved "nucleophile-acid-histidine" catalytic triad. In summary, this study showed that intracellular proteases were responsible for the reduced yields of heterologous proteins and provided an efficient strategy to enhance the extracellular production of AHL lactonase AiiO-AIO6.
\end{abstract}

Keywords: AHL lactonase, Bacillus subtilis, Quorum quenching, Intracellular protease-deletion mutant

\section{Introduction}

Quorum quenching (QQ) blocks bacterial cell-to-cell communication (i.e., quorum sensing), and is a promising antipathogenic strategy to control bacterial infection via inhibition of virulence factor expression and biofilm formation. QQ strategy has been applied to many fields

\footnotetext{
*Correspondence: yangyalin@caas.cn; zhouzhigang03@caas.cn ${ }^{1}$ Sino-Norwegian Fish Gastrointestinal Microbiota Joint Lab, Feed Research Institute, Chinese Academy of Agricultural Sciences, Beijing 100081, China

${ }^{2}$ Key Laboratory for Feed Biotechnology of the Ministry of Agriculture, Feed Research Institute, Chinese Academy of Agricultural Sciences, Beijing 100081, China

Full list of author information is available at the end of the article
}

such as aquaculture, crop production and anti-biofouling (Grandclément et al. 2016). QQ enzyme AiiO-AIO6 from Ochrobactrum sp. M231 has excellent properties such as high ion and chemical resistance, high thermostability, broad-spectrum substrate specificity and high enzyme activity, and shows biotherapeutic potential against important bacterial pathogens of aquatic organisms (Zhang et al. 2011). AiiO-AIO6 can be secretory expressed in Bacillus subtilis via a non-classical secretion pathway (Pan et al. 2016). However, the secretion level of AiiO-AIO6 in B. subtilis is low and needs to be improved.

Host proteases have been considered as one of the major factors limiting the production of heterologous 
proteins in B. subtilis. Many studies have showed that the deletions of protease genes have improved the yields of many recombinant proteins, such as the use of proteasedeficient strains to enhance extracellular pullulanase production in B. subtilis (Zhang et al. 2018). However, these studies have focused on knocking out membrane-bound, cell wall-associated or secreted protease genes; few studies have involved the deletion of intracellular proteases. $B$. subtilis encodes three proteases (HtrA, HtrB and WprA) that are known to be functional at the wall/membrane interface or in the wall itself (quality control proteases), and seven proteases (AprE, Bpr, Epr, Mpr, NprB, NprE and $\mathrm{Vpr}$ ) that are secreted into the culture medium (feeding proteases). Previous work has shown that some or all of these proteases were responsible for the reduced yields of various heterologous proteins (Westers et al. 2008; Wu et al. 1993, 2002). Intracellular proteases also play an important role in quality control and act as a major barrier to the production of certain secreted recombinant proteins (Molière and Turgay 2009; Park and Schumann 2015; Westers et al. 2004b). For example, an intracellular protease such as AprX was involved in degradation of a heterologous protein during the late stationary growth phase and the AprX mutant exhibited enhanced production of heterologous proteins (Kodama et al. 2007).

The aim of this study was to compare and evaluate the effect of these intracellular proteases such as serine protease (TepA), cysteine protease (YwpE), metalloproteinase $(\mathrm{YmfH})$ and unknown protease $(\mathrm{YrrN})$, on the secretion of AiiO-AIO6 by B. subtilis. The degradation mechanism and homology of AiiO-AIO6 was also analyzed by LC-MS/MS and phylogenetic tree.

\section{Materials and methods}

\section{Bacterial strains, plasmids and growth conditions}

The bacterial strains and plasmids used in this study are described in Additional file 1: Tables S1 and S2. All mutated B. subtilis strains were derivatives of $B$. subtilis strain 1A751. All B. subtilis strains were grown in super-rich medium containing $25 \mathrm{~g}$ Bacto tryptose, $20 \mathrm{~g}$ Bacto yeast extract and $3 \mathrm{~g} \mathrm{~K}_{2} \mathrm{HPO}_{4}$ per liter (pH 7.5) or agar plates with ampicillin $(100 \mu \mathrm{g} / \mathrm{mL})$, spectinomycin $(100 \mu \mathrm{g} / \mathrm{mL})$, zeocin $(25 \mu \mathrm{g} / \mathrm{mL})$ and kanamycin $(25 \mu \mathrm{g} /$ $\mathrm{mL})$.

\section{Construction of intracellular protease deletion mutants}

The primers used in this study are summarized in Additional file 1: Table S3. To create the gene deletion loci for tepA, $y m f H, y r r N$ and $y w p E$, the genes and flanking regions (about $2000 \mathrm{bp}$ ) were amplified from chromosomal DNA using their respective upstream and downstream primers. These PCR products were ligated into $\mathrm{T}$-vectors to generate template vectors $\mathrm{pT}$-tepA,
pT-ymfH, pT-yrrN, and pT-ywpE, which were then used as templates to amplify the $5^{\prime}$ and $3^{\prime}$ flanks of these genes. pPIC9K was used as template to amplify the zeocin resistance gene. The zeocin resistance gene replaced the deletion gene and was inserted between the $5^{\prime}$ flanks of the deletion gene and the $3^{\prime}$ flanks of the deletion gene of template vectors to generate antibiotic selection marker knockout vectors $\mathrm{p} \Delta t e p A, \mathrm{p} \Delta y m f H, \mathrm{p} \Delta y r r N$ and $\mathrm{p} \Delta y w p E$. Knockout vectors were transformed to $B$. subtilis 1A751. The suspect mutant cells resistant to zeocin were further identified by diagnostic PCR with the upstream forward primer/the downstream reverse primer of these deletion genes and the upstream forward primer of $5^{\prime}$ flanks of these deletion genes/the downstream reverse primer of zeocin gene. The mutant was further confirmed by DNA sequencing.

\section{Secretory expression of AiiO-AIO6}

The AiiO-AIO6 expression plasmid pWB-AIO6BS was constructed following protocols as described previously (Pan et al. 2016). pWB-AIO6BS was transformed into the B. subtilis $1 \mathrm{~A} 751$ and its four intracellular protease gene deletion derivatives. The secretion of AiiO-AIO6 from $B$. subtilis was studied using pWB-AIO6BS-harboring strains $1 \mathrm{~A} 751, \mathrm{BS} \Delta t e p A, \mathrm{BS} \Delta y m f H, \mathrm{BS} \Delta y r r N$, and BS $\triangle y w p E$. B. subtilis cells were cultured in SR medium with kanamycin $(25 \mu \mathrm{g} / \mathrm{mL})$ at $200 \mathrm{rpm}$ for $24 \mathrm{~h}$ at $30^{\circ} \mathrm{C}$. Bacterial growth was monitored by measuring optical density at $600 \mathrm{~nm}$ with the BioPhotometer plus of Eppendorf AG (Hamburg, Germany). Culture supernatant was separated from $B$. subtilis culture by centrifugation at $12,000 \mathrm{~g}\left(10 \mathrm{~min}, 4{ }^{\circ} \mathrm{C}\right)$ and subjected to AHL-lactonase activity bioassay. Proteins in the supernatants were precipitated with two volume of ice-cold acetone, and then acetone precipitations were separated on $12 \%$ polyacrylamide (TGX Stain-Free FastCast Acrylamide Kit, BioRad) and transferred to polyvinylidene difluoride (PVDF) membranes (Immobilon; $0.45 \mu \mathrm{m}$ pore size; Millipore). All stain-free gels were imaged with the Gel Doc XR+ documentation system (Bio-Rad). Western blot analysis was carried out using monoclonal mouse-anti-His-Tag antibody (TianGen, China) as the primary antibody and performed using One Step Western Kit HRP (mouse) according to the manufacturer's instructions (CW Biotech Company Beijing, China).

\section{Enzyme assays}

One unit of AHL lactonase activity was defined as the amount of enzyme that hydrolyzed 1 nmol 3-oxo- $\mathrm{C}_{8}$ HSL per minute. For the hydrolysis assay, the reaction mixture $(500 \mu \mathrm{L})$ contained $50 \mu \mathrm{L}$ AiiO-AIO6, $0.248 \mathrm{mM}$ 3-oxo-C8-HSL, and $10 \mathrm{mM}$ PBS (pH 7.0). The reaction was terminated at $70{ }^{\circ} \mathrm{C}$ for $10 \mathrm{~min}$ after the mixture was 
incubated at $30{ }^{\circ} \mathrm{C}$ for $30 \mathrm{~min}$. The reaction mixtures were centrifuged at $10,000 \mathrm{~g}$ for $10 \mathrm{~min}$ at $4{ }^{\circ} \mathrm{C}$ and the supernatants after $0.22 \mu \mathrm{m}$ filtration were used for HPLC analysis and quantification. The residual 3-oxo-C8-HSL was separated in a Shimadzu InertSustain C18 column at $30{ }^{\circ} \mathrm{C}$ with a constant flow rate of $1 \mathrm{~mL} / \mathrm{min}$ in isocratic elution with aqueous-organic mobile phase containing $0.375 \%$ triethylamine/acetonitrile $(64: 36, \mathrm{~V} / \mathrm{V})$, and then detected with an UV/visible light detector (waters) at $201 \mathrm{~nm}$. The remaining AHLs were quantified by calculating the peak areas for a given retention time compared to AHL solutions of known concentrations. Furthermore, for the control, AiiO-AIO6 was replaced with inactivated AiiO-AIO6 by heat treatment $\left(100{ }^{\circ} \mathrm{C}\right.$ for $\left.5 \mathrm{~min}\right)$. All determinations were performed in four replicates.

An enzyme assay was carried out using assay systems that consisted of mixtures of PBS ( $\mathrm{pH} 7.0)$ and 0.249 to $0.870 \mathrm{nM}$ 3-oxo-C8-HSL and an incubation time of $30 \mathrm{~min}$ at $30^{\circ} \mathrm{C}$, and the corresponding reaction rate was calculated. The Michaelis-Menten equation in GraphPad Prism 8 was used to measure the Vmax, $\mathrm{Km}$ and Kcat of Michaelis-Menten kinetics.

\section{LC-MS/MS analysis of the hydrolysis products of AHL by AiiO-AIO6}

The above hydrolysis products of AHL by purified AiiOAIO6 were extracted three times with ethyl acetate. The combined organic phase was then evaporated to dryness. The samples were dissolved in methanol and separated by a 50 -min isocratic elution (mobile phase: methanol: water (60:40; v/v); flow rate: $0.25 \mathrm{~mL} / \mathrm{min}$ ) on a Thermo Scientific Dionex Ultimate 3000 UPLC system with C18 column. MS experiments were conducted on a Thermo $\mathrm{Q}$ Exactive mass spectrometer (Thermo Fisher Scientific, San Jose, CA, USA) in a data-dependent acquisition mode with mass range 50 to $750 \mathrm{~m} / \mathrm{z}$ using the Xcalibur 2.1.2 software followed by ten data-dependent MS/MS scans. The full scan and fragment spectra were collected with resolutions of 70,000 and 17,500 respectively.

\section{Phylogenetic analysis}

For AHL lactonase AiiO-AIO6, homologous protein sequences were retrieved from NCBI after identification by BLAST. Multiple sequence alignment was constructed using DNAMAN. The phylogenetic tree was generated by the neighbor-joining method with the ClustalW (MEGA7).

\section{Results}

Identification of intracellular protease-deficient $B$. subtilis strains

In order to study the effects of intracellular proteases on AiiO-AIO6 secretion expression, four intracellular proteases, TepA, YmfH, YrrN and YwpE, were deleted from the genome of $B$. subtilis 1A751, using the knockout vectors $\mathrm{p} \Delta t e p A, \mathrm{p} \Delta y m f H, \mathrm{p} \Delta y r r N$, and $\mathrm{p} \Delta y w p E$, respectively. First, specific primers of knockout genes were used, no products were amplified in mutant strains which confirmed the successful knocked out. Then the resulting B. subtilis knockout strains (BS $\Delta t e p A, \mathrm{BS} \Delta y m f H$, BS $\triangle y r r N$ and $B S \triangle y w p E$ ) were confirmed by PCR with the upstream forward primer of $5^{\prime}$ flanks of these deletion genes and the downstream reverse primer of zeocin resistance genes. No product was amplified from the wild-type strain, while PCR products with expected sizes were amplified from intracellular protease-deficient B. subtilis strains using their respective primer pairs (Fig. 1b).

\section{Secretory production of AiiO-AIO6 in intracellular protease-deficient $B$. subtilis strains and its kinetic characterization}

Studies showed that the acidic group of SDS binds to the reversed-phase column where it serves as an ion

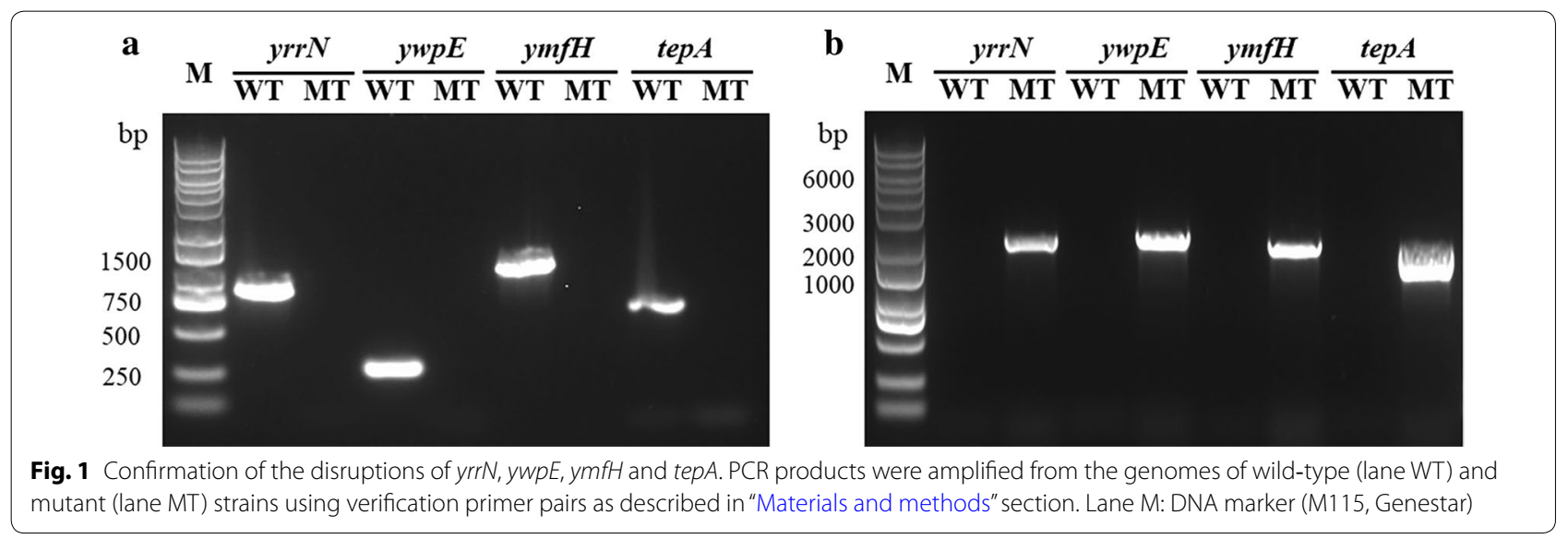


exchanger and can interfere with RPLC. To avoid the interference of SDS in the separation of AHL by HPLC, we inactivated enzyme reaction by heat treatment. To determine the optimal temperature at which AiiOAIO6 was inactive and 3-oxo-C8-HSL was still stable, thermal inactivation of AiiO-AIO6 was measured. The inactivation rate of AiiO-AIO6 was $66.50 \%, 99.20 \%$, 99.97\%, 100\% (Fig. 2a) after heating at 60, 70, 80, and $100{ }^{\circ} \mathrm{C}$ for $10 \mathrm{~min}$, respectively. Then AHL after treatment at 30,70 and $80{ }^{\circ} \mathrm{C}$ for 10 min was detected by HPLC and the remaining amount of AHL were calculated as $100 \%, 96.49 \%$ and $85.62 \%$ (Fig. 2b), respectively. Therefore, the optimal inactive temperature for enzyme reaction was $70{ }^{\circ} \mathrm{C}$ (Additional file 1: Tables S4, S5).
By detecting the growth of bacteria, we found that these mutations did not affect bacterial growth of strain 1A751 which compared with the wild-type (pWB980AIO6BS/BS1A751) (Fig. 3). The culture supernatants from the wild-type strain and mutant strains were analyzed by SDS-PAGE and Western blotting. SDS-PAGE clearly showed that the band intensities of AiiO-AIO6 from the recombinant intracellular protease-deficient strains were significantly higher than that observed in the wild-type B. subtilis 1A751 strain (Fig. 4a). Moreover, the AiiO-AIO6 activities in culture supernatants of recombinant mutant strains were significantly higher than that of the recombinant wild-type strain. Recombinant strain $\mathrm{BS} \triangle y w p E / \mathrm{pWB}-\mathrm{AIO} 6 \mathrm{BS}$ achieved the highest secretion of $1416.47 \mathrm{U} / \mathrm{mL}^{-} \mathrm{OD}_{600}$ at $24 \mathrm{~h}$, which was about
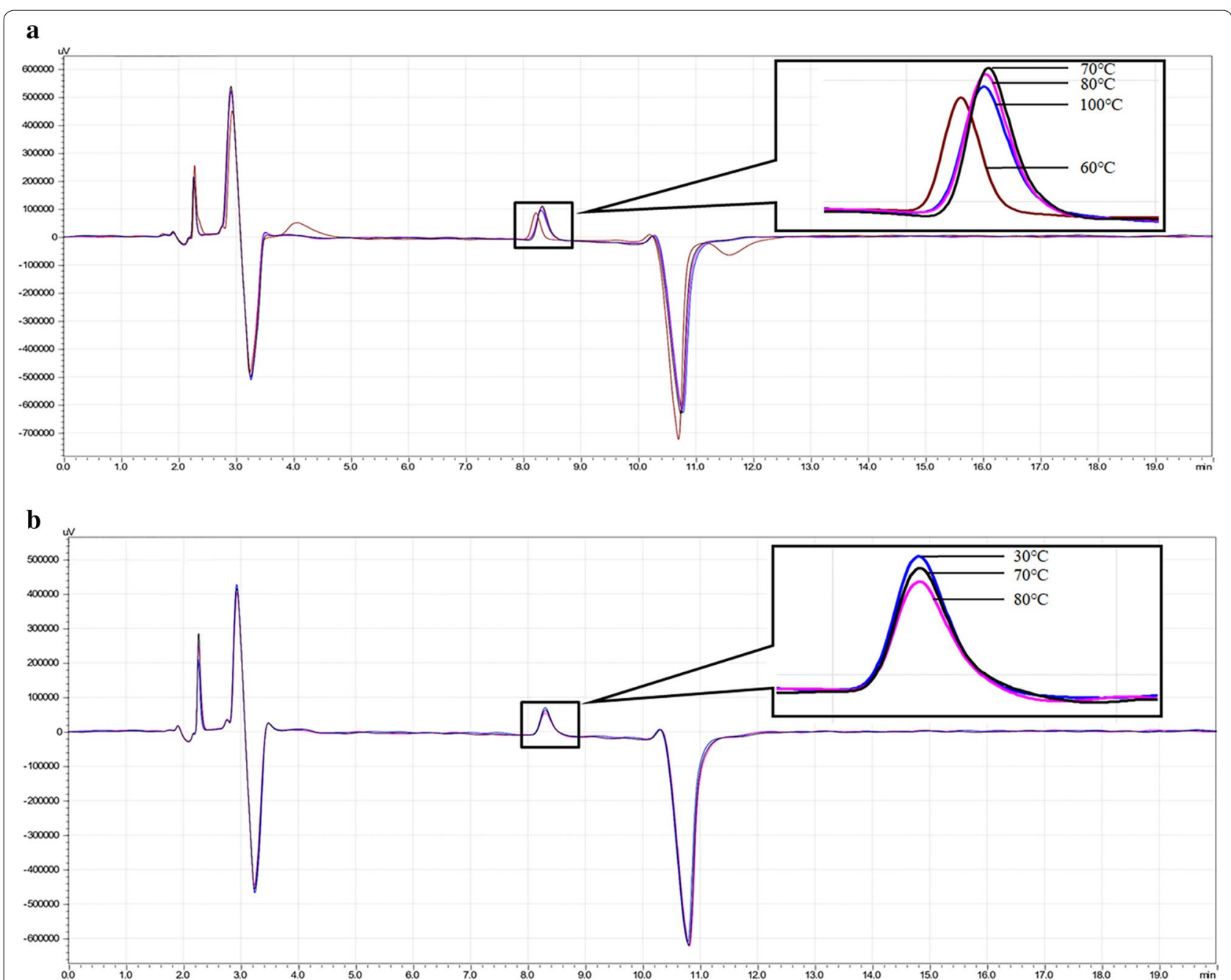

Fig. 2 The inactivation of AiiO-AIO6 and the stability of 3-oxo-C8-HSL under different temperatures. a AiiO-AIO6 was heated at 60, 70, 80, and $100{ }^{\circ} \mathrm{C}$ (as control with $100 \%$ inactivation rate) for $10 \mathrm{~min}$ and then reacted with 3 -oxo-C 8 - HSL at $30{ }^{\circ} \mathrm{C}$ for 20 min. The reaction products were analyzed by HPLC to quantify 3-oxo-C8-HSL. b 3-oxo-C8-HSL solution was treated at 30 (as control with $0 \%$ lactonolysis rate), 70 and $80{ }^{\circ} \mathrm{C}$ for 10 min then detected directly by HPLC to quantify 3-oxo-C8-HSL 


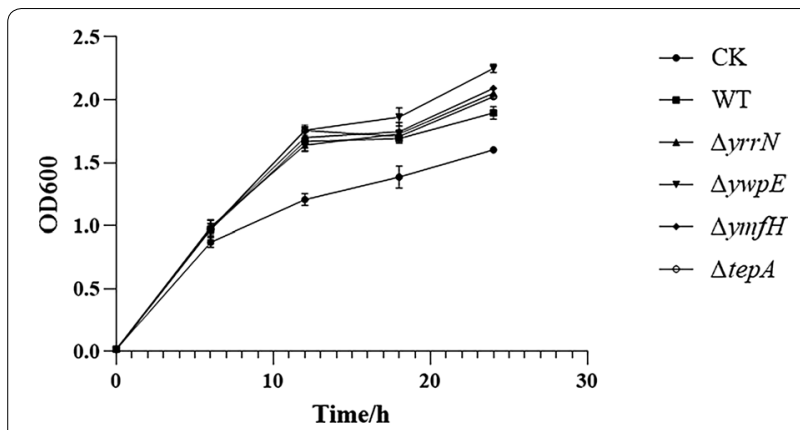

Fig. 3 Growth curve of intracellular protease-deficient strains. CK, pWB980/BS1A751, wild-type (WT), pWB980-AIO6BS/BS1A751; $\triangle y m f H, \triangle y r r N, \triangle y w p E, \triangle t e p A$ : intracellular protease-deficient mutants harboring the expression vector pWB980-AIO6BS

$121 \%$ higher than that of the recombinant wild-type strain (Fig. 4b). Under optimal conditions ( $\mathrm{pH} 7.0,30^{\circ} \mathrm{C}$, 3-oxo-C8-HSL as substrate), the specific activity of purified AiiO-AIO6 was $4.41 \times 10^{3} \mathrm{U} / \mathrm{mg}$. Kinetic parameters including $\mathrm{Km}$, Vmax, and Kcat values of AiiO-AIO6 were calculated as $0.4151 \mathrm{mmol} / \mathrm{L}, 16,949 \mathrm{nmol} / \mathrm{mg} / \mathrm{min}$, and $9.34 \mathrm{~S}^{-1}$, respectively.

\section{AlO6 is an AHL lactonase}

To identify which QQ enzyme AiiO-AIO6 belongs to, the degradation products of 3-oxo-C8-HSL by purified AiiOAIO6 were analyzed using LC-MS/MS. As shown in Fig. 5, LC-MS/MS analysis of the 14.44 min HPLC fraction showed a $\mathrm{M}-\mathrm{H}$ ion at a mass-to-charge ratio $(\mathrm{m} / \mathrm{z})$ of 258.1, which was identical to that of the open-ring 3-oxo-C8-HSL, and tandem MS of the precursor ion at a $m / z$ of 258.1 showed a characteristic fragment at a $m / z$ of 118.0, corresponding to homoserine, resulting from the lactone-opened $\mathrm{N}$-(3-oxooctanoyl)-L-homoserine. These results strongly suggested that AiiO-AIO6 was an AHLlactonase which hydrolyzes the lactone ring of AHLs.

Phylogenetic analysis of known AHL-lactonase indicated that AiiO-AIO6 was classified as an $\alpha / \beta$ hydrolase family member (Fig. 6). AiiO-AIO6 shared 84.8\%, 13.8\%, $12.7 \%$ and $10.8 \%$ amino acid sequence identity with AidH from Ochrobactrum sp. Strain T63 (Mei et al. 2010), Aii810 from metagenome (Kawasaki and Suzuki 1990), AiiM from M. testaceum StLB037 (Wang et al. 2010) and AidA from Acinetobacter baumannii (López et al. 2017), respectively. Like AidH, AiiO-AIO6 also contained the "nucleophile-acid-histidine" catalytic triad (Ser100, His246 and Glu214) that was conserved among members of the alpha/beta-hydrolase family and was metal-independent AHL-lactonase (Gao et al. 2013) (Fig. 7).

\section{Discussion}

Bacillus subtilis has a strong ability to secrete natural or homologous proteins. About $60 \%$ of commercially available proteins are produced by Bacillus, but the expression types and products of heterogenous proteins are still limited (Fu et al. 2007). Two alternative strategies to improve heterologous protein secretion production are to screen functional signal peptides (Kang et al. 2014) and to delete host proteases to reduce proteolytic degradation
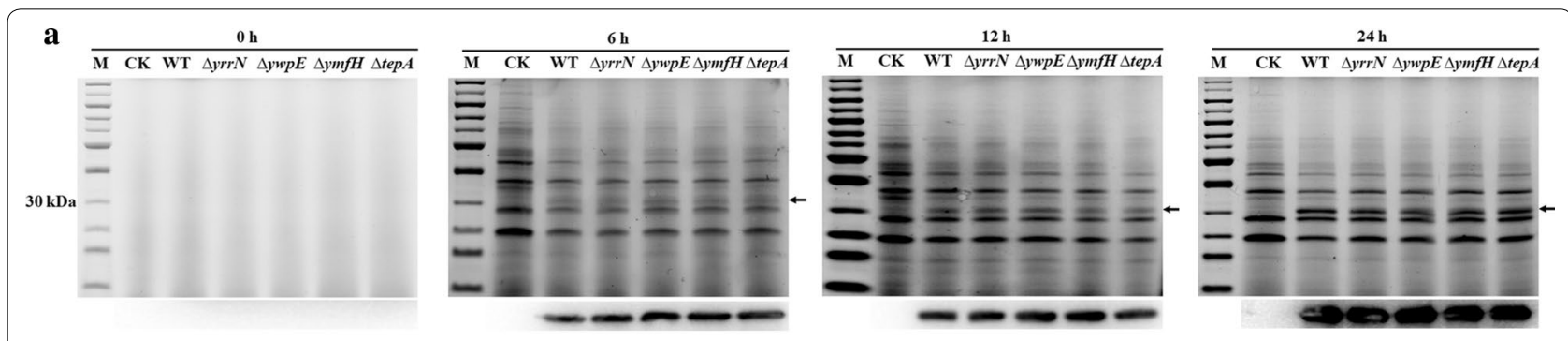

b
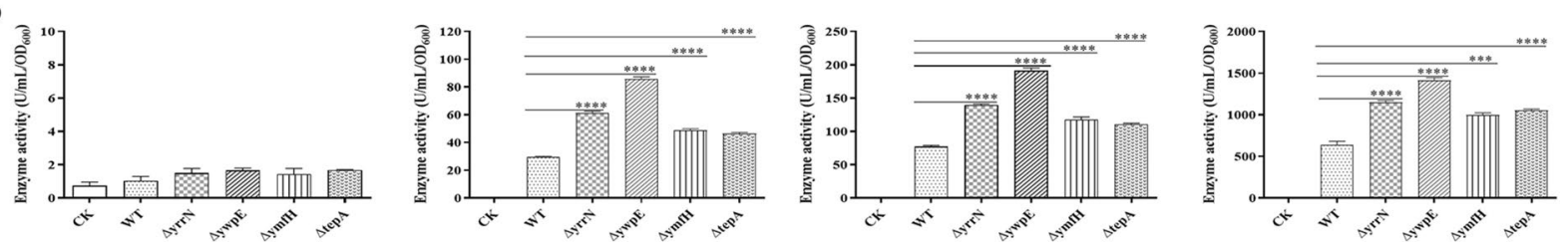

Fig. 4 Secretion of AiiO-AlO6 by intracellular protease-deficient strains. a SDS-PAGE (up) and Western blotting (down) of AiiO-AlO6 secreted in culture medium derived from wild-type (WT) strain $1 \mathrm{~A} 751$ or intracellular protease-deficient mutants after different time points cultivation at $37^{\circ} \mathrm{C}$. M, protein marker; CK, pWB980/BS1A751, wild-type (WT), pWB980-AIO6BS/BS1A751; $\triangle y m f H, \Delta y r r N, \Delta y w p E, \Delta t e p A$ : intracellular protease-deficient mutants harboring the expression vector pWB980-AlO6BS. b Secreted AiiO-AlO6 activity present in wild-type or intracellular protease-deficient mutant culture medium. $p$ values were calculated using unpaired two-tailed T-tests. An asterisk indicates a statistically significant difference between two activity values $(p<0.05)$ 


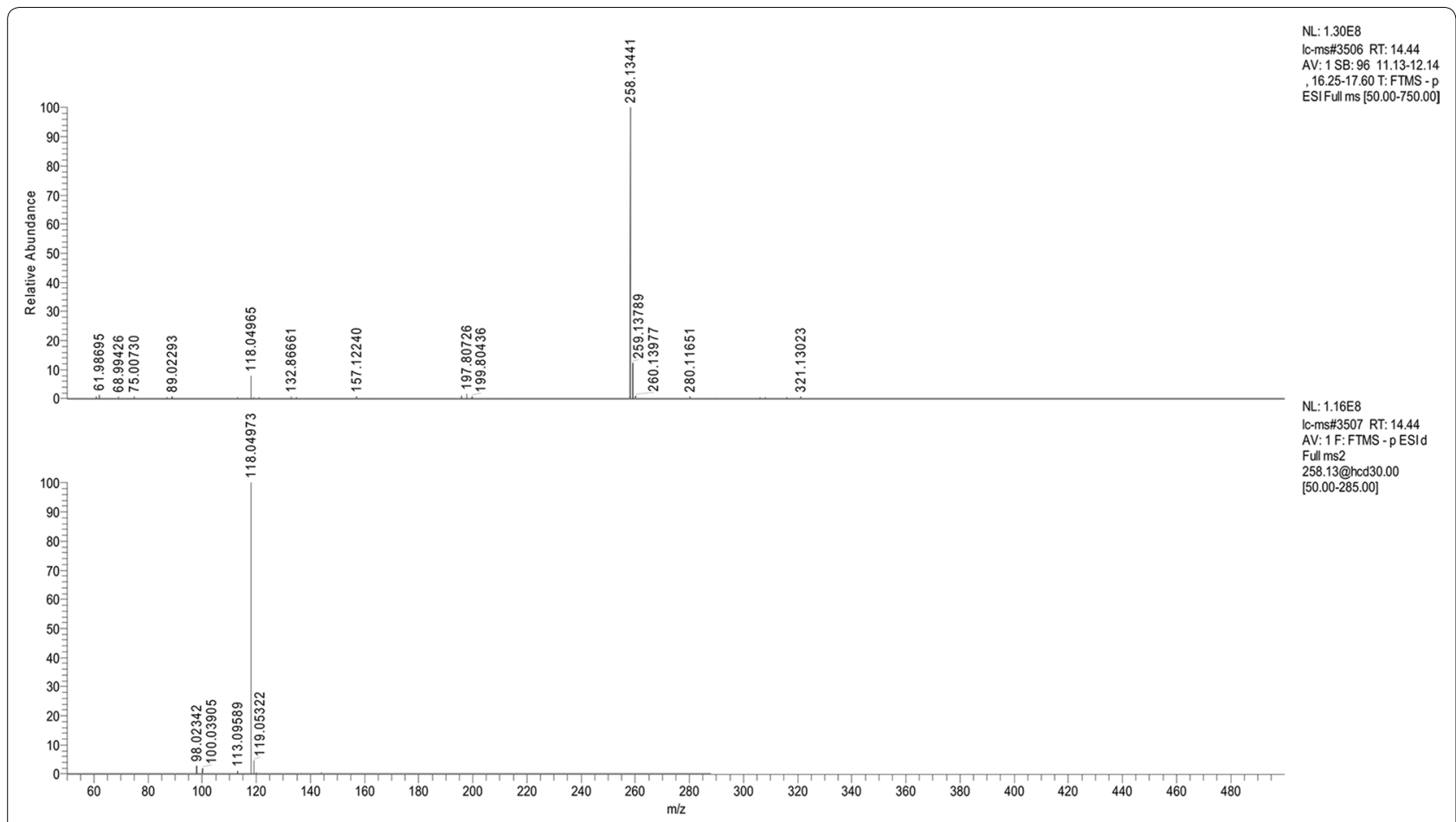

Fig. 5 LC-MS/MS analysis of the hydrolysis product of 3-oxo-C8-HSL by AiiO-AIO6. MS ${ }^{1}$ analysis of the 14.44 min HPLC fragment of enzymatic hydrolysates showed a mainprecursor $\left(\mathrm{M}-\mathrm{H}\right.$ ) ion at $\mathrm{m} / \mathrm{z}$ of 258.1 (upper panel). $\mathrm{MS}^{2}$ spectra of the precursor ion at $\mathrm{m} / \mathrm{z}$ of 258.1 by tandem mass spectrometry showed a main fragment ion at $\mathrm{m} / \mathrm{z} 118.1$ (bottom panel)

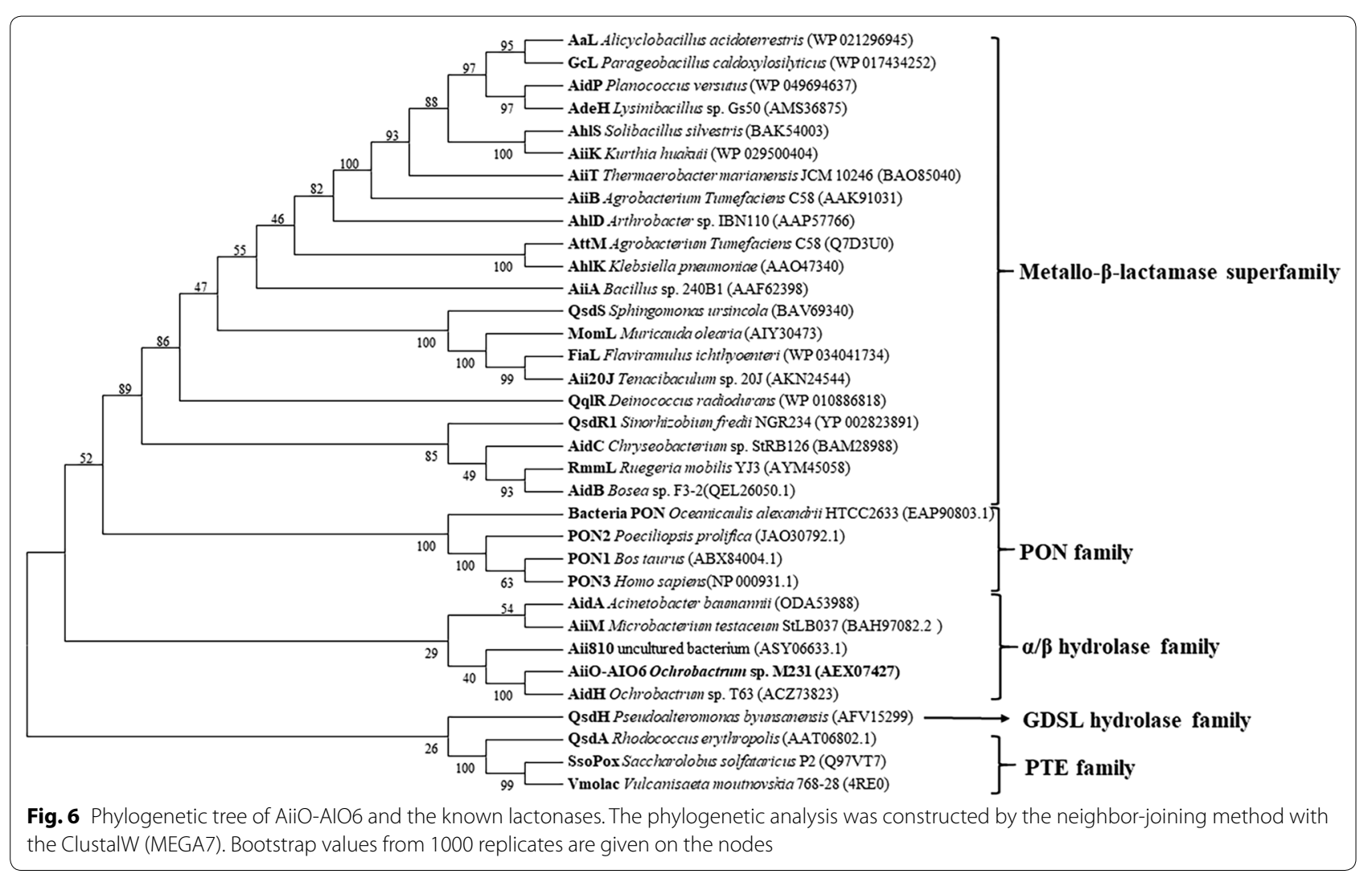




Aiio-AIO6
AidH
AidA
Aii810
AiiM

AiIO-AIO6

AidH

AidA

Aii 810

Aiim

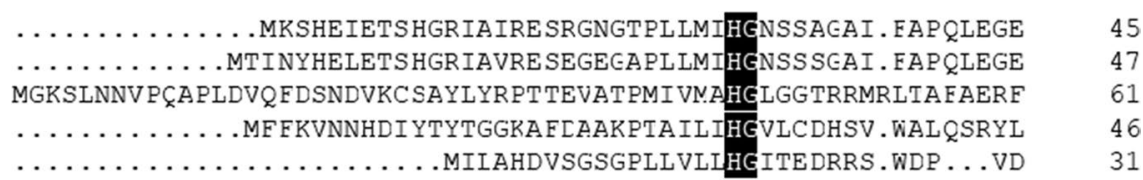

Nucleophile elbow

Aiio-AIO6 GHIGIEMISREPGMRGLM........ ITGTPPVA....REEVGQGERSGPDNAIAGQEAFS AidH GHIGIEMIARYPEMRGLM........ITGTPPVA...REEVGQGEKSGPDNAIAGQEIFS AidA GGHVIATAANDNRIAAVISQCPETDGFSSSNAMNPITTLKLMGIALKDKIGSILGAKPVMV Aii 810 SLIAMEAAATLKDRISHL . . . . . . VLVGTAF P.MKVS PALLESALHTPECุAIAMVNTFS Aiim GIVATAYGALFFARAIVN....... VDQPLQLAGMQGQV

\section{2}

104

122

99

90

Aiio-AIO6
AidH
AidA
Aii810
AiiM

AiiO-AIO6
AidH
AidA
Aii810
AiiM

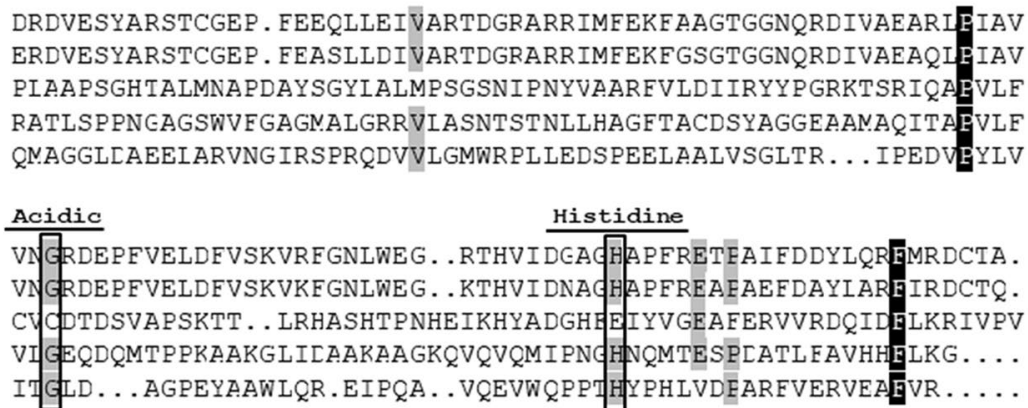

Fig. 7 Comparison of amino acid sequences of AiiO-AIO6 and several alpha/beta-hydrolases. The alignment was generated by DNAMAN. Identities are highlighted in white with a black background, and similarities are shaded gray. The catalytic triad residues are boxed with rectangles. The amino acid residues essential for AHL-degrading activity are indicated by asterisks. AiiO-AlO6, AiiO-AlO6 from Ochrobactrum sp. M231 (GenBank accession no. AEX07427); AidH, AidH from Ochrobactrum sp. T63 (GenBank accession no. GQ849010); AidA, AidA from Acinetobacter baumannii (GenBank accession no. ODA53988); Aii810, Aii810 from uncultured bacterium (GenBank accession no. ASY06633.1); AiiM, AiiM from Microbacterium testaceum StLB037 (GenBank accession no. BAJ75775)

(Westers et al. 2004b). Since AiiO-AIO6 was a protein secreted through a non-classical pathway, a conventional signal peptide can not improve its secretion (Pan et al. 2016).

Many studies have focused on engineering of extracellular proteases to reduce extracellular proteolysis. There are few reports on whether deletion of intracellular protease could improve the secretion production of heterogenous protein, so we have tried to use an intracellular protease-deletion strain to improve its secretion. In this study, four intracellular proteases were deleted in order to study their effect on the secretion of AiiO-AIO6. YwpE is one of two putative sortase homologues of $B$. subtilis. YwpE encodes a protein of 102 amino acids with the LxTC motif for the sortase activity at the C-terminus, but lack of a transmembrane anchor at its $\mathrm{N}$-terminus. The study of the role of YwpE in displaying two potential sortase substrates YhcR and YfkN on the cell wall showed that YwpE seems not to play a major role, if any, as a sortase (Nguyen et al. 2011). The peptidase U32 protein YrrN is one member of the yrrMNO operon which is important for the biosynthesis of 5-hydroxyuridine, YrrN is involved in the synthesis of 5-methoxyuridine (Nguyen et al. 2011). The cytoplasmic ClpP-like germination protease TepA is involved in spore outgrowth in B. subtilis, and is not proposed to be a signal peptide peptidase but to degrade a specialized family of small DNA-binding proteins during the process of spore outgrowth (Traag et al. 2013; Westers et al. 2004a). YmfH is identified by the presence of Peptidase_M16 and Peptidase_M16_C domains, and is predicted to encode uncharacterized zinc protease with unknown specificity (Hummels et al. 2017). In this study, the deletion of any of the four proteases improved the secretion of AiiO-AIO6, but the degree of improvement was different. Their effect on the secretion of AiiO-AIO6 in B. subtilis was YwpE > YrrN > TepA or $\mathrm{YmfH}$, which may be due to the difference in the degradation ability of these proteases to AiiO-AIO6 or the 
degradation ability of the proteins assisting the secretion of AiiO-AIO6. This study showed that deletion of intracellular protease is also a good strategy to improve the secretion and expression of heterogenous protein.

A simple and accurate method for monitoring enzymatic activity is of fundamental importance to the study of QQ enzymes. At present, the main detection methods for QQ enzyme activity are microbiosensor-based biological detection and chromatography-based physicochemical detection (mainly HPLC) (Liu et al. 2018). Of these, the physicochemical detection method is quantitatively more accurate but requires sample pretreatment. The main method for extracting AHLs from aqueous samples is liquid-to-liquid extraction (Feng et al. 2014; Tan et al. 2014). However, the extractant and extraction steps will affect the extraction efficiency, and there are some problems such as hazardous organic solvent consumption and cumbersome and time-consuming experimental steps. The composition of the enzyme reaction solution is not too complex, so we tried to bypass the extraction step to directly carry out HPLC.

The HPLC method is an efficient method for quantifying AHLs, but QQ enzymes show low sensitivity to most metal ions and chemicals, and AHLs are sensitive to $\mathrm{pH}$ and temperature (Yates et al. 2002). Most enzymes such as AiiA (Yates et al. 2002), AiiK (Dong et al. 2018), AidF (Fan et al. 2020) and AiiO-AIO6 can be inhibited by sodium dodecyl sulfate (SDS) (Zhang et al. 2011), so SDS is usually used as an inhibitor to stop the enzyme reaction (Wang et al. 2004). The strongly acidic group of SDS can bind to the reversed-phase column where it will serve as an ion exchanger and can interfere with RPLC separation (Kawasaki and Suzuki 1990), which seriously affects the HPLC detection. Therefore, it is necessary to find an effective method to terminate the enzyme reaction. In this study, we found that $10 \mathrm{~min}$ treatment at $70{ }^{\circ} \mathrm{C}$ is an effective way to inactivate AiiO-AIO6 and has little effect on the stability of the signal molecule. This method eliminates the extraction process and greatly simplifies the detection of enzyme activity by HPLC. The extraction-eliminated HPLC improved the detection efficiency of QQ enzyme activity.

AiiO-AIO6 and AidH are both members of the $\alpha / \beta$ hydrolase superfamily, and they do not contain a metal binding motif. Although there is a high sequence similarity between AiiO-AIO6 and AidH, our previous study found that there are some differences between AiiOAIO6 and AidH in their characterization. We showed that EDTA and various ions (including $\mathrm{Mn}$ ions) did not affect the activity of AiiO-AIO6, and the activity of AiiO-AIO6 did not need metal ions (Zhang et al. 2011), which was also consistent with the metal free binding motif. However, manganese ion was very important for the activity of AidH (Mei et al. 2010). Both AiiO-AIO6 and AidH have a broad substrate spectrum; they can both degrade C6-HSL to C10-HSL, 3-oxo-C6-HSL to 3-oxoC12-HSL and 3-hydroxy-C12-HSL. However, the substrate specificity of AiiO-AIO6 is different from that of AidH. AidH has a broad substrate specificity, and its degradation activity for different substrates showed no significant difference (Gao et al. 2013). However, AiiO-AIO6 has narrow substrate specificity. AiiO-AIO6 exhibited high activity to 3-oxo-C8-HSL but greatly reduced activities to 3-oxo-C6-HSL, C6-HSL, C12-HSL and 3-hydroxyC12-HSL (Zhang et al. 2011). These differences in their characterization should be due to the difference between their amino acid sequences.

In summary, this study improves the secretion production of AiiO-AIO6 by engineering host intracellular protease, and YwpE protease knockout will be more conducive for secretion of AiiO-AIO6. Termination of enzyme reaction by temperature eliminated the sample extraction, so HPLC without the extraction step showed improved detection efficiency of QQ enzyme activity. AiiO-AIO6 is a lactonase of the $\alpha / \beta$ hydrolase superfamily. This study provides an alternative strategy for improving the secretion of heterologous protein by Bacillus, which will contributes to the study of quenching enzyme properties, and promote the application of AiiOAIO6 in the disease control of Gram-negative bacteria in aquaculture.

\section{Supplementary information}

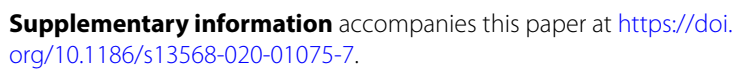

Additional file 1: Table S1. Bacterial strains. Table S2. Plasmids. Table S3. Primers. Table S4. The remaining amount of AHL under different temperatures. Table S5. The inactivation rate of AiiO-AlO6 under different temperatures.

\section{Abbreviations}

QQ: Quorum quenching; PCR: Polymerase chain reaction; SDS-PAGE: Sodium dodecylsulfate polyacrylamide gel electrophoresis; PBS: Phosphate buffer solution.

\section{Authors' contributions}

Participated in research design: $Y Y$ and $Z Z ;$ Conducted experiments and performed data analysis: $R X, Y Y, X P, C G, X L, F Z, J H, C R, Z Z$ and $Z Z$; Wrote the manuscript or contributed to the manuscript: $R X, Y Y, Y Y, T T, J L C$ and $Z Z$. All authors read and approved the final manuscript.

\section{Funding}

This work was supported by the key project of the National Natural Science Foundation of China (Grant No. 31672294, 31872584), the Beijing Earmarked Fund for Modern Agro-industry Technology Research Systems (SCGWZJ 20201104-4), the Innovation Capability Support Program of Shaanxi (2018TD021), and the Se-enriched Special Science and Technology Project of China Institute of Se-enriched Industry (2018FXZX02-04).

Availability of data and materials

All the data are presented in the main paper or the additional information. 
Ethics approval and consent to participate

No animal or human subjects were used in this work.

\section{Consent for publication}

Not applicable.

\section{Competing interests}

The authors declare that they have no competing interests.

\section{Author details}

${ }^{1}$ Sino-Norwegian Fish Gastrointestinal Microbiota Joint Lab, Feed Research Institute, Chinese Academy of Agricultural Sciences, Beijing 100081, China.

${ }^{2}$ Key Laboratory for Feed Biotechnology of the Ministry of Agriculture, Feed Research Institute, Chinese Academy of Agricultural Sciences, Beijing 100081 ,

China. ${ }^{3} \mathrm{NIBIO}$, Norwegian Institute of Bioeconomy Research, 1431 Ås, Norway.

Received: 18 April 2020 Accepted: 28 July 2020

Published online: 05 August 2020

\section{References}

Dong WW, Zhu J, Guo X, Kong DL, Zhang Q, Zhou YQ, Liu LY, Zhao SM, Ruan ZY (2018) Characterization of AiiK, an AHL lactonase, from Kurthia huakui LAM0618T and its application in quorum quenching on Pseudomonas aeruginosa PAO1. Sci Rep 8(1):6013

Fan X, Ye T, Li Q, Bhatt P, Zhang L, Chen S (2020) Potential of a quorum quenching bacteria isolate Ochrobactrum intermedium D-2 against soft rot pathogen Pectobacterium carotovorum subsp. carotovorum. Front Microbiol 11:898

Feng $H$, Ding $Y$, Wang $M$, Zhou G, Zheng $X$, He H, Zhang $X$, Shen $D$, Shentu $J$ (2014) Where are signal molecules likely to be located in anaerobic granular sludge? Water Res 50:1-9

Fu LL, Xu ZR, Li WF, Shuai JB, Lu P, Hu CX (2007) Protein secretion pathways in Bacillus subtilis: implication for optimization of heterologous protein secretion. Biotechnol Adv 25(1):1-12

Gao A, Mei GY, Liu S, Wang P, Tang Q, Liu YP, Wen H, An XM, Zhang LQ, Yan XX (2013) High-resolution structures of AidH complexes provide insights into a novel catalytic mechanism for $\mathrm{N}$-acyl homoserine lactonase. Acta Crystallogr D Biol Crystallogr 69(1):82-91

Grandclément C, Tannières M, Moréra S, Dessaux Y, Faure D (2016) Quorum quenching: role in nature and applied developments. FEMS Microbiol Rev 40(1):86-116

Hummels KR, Witzky A, Rajkovic A, Tollerson R, Jones LA, Ibba M, Kearns DB (2017) Carbonyl reduction by Ymfl in Bacillus subtilis prevents accumulation of an inhibitory EF-P modification state. Mol Microbiol 106(2):236-251

Kang Z, Yang S, Du G, Chen J (2014) Molecular engineering of secretory machinery components for high-level secretion of proteins in Bacillus species. J Ind Microbiol Biotechnol 41(11):1599-1607

Kawasaki H, Suzuki K (1990) Separation of peptides dissolved in a sodium dodecyl sulfate solution by reversed-phase liquid chromatography: removal of sodium dodecyl sulfate from peptides using an lon-exchange precolumn. Anal Biochem 186(2):264-268

Kodama T, Endo K, Sawada K, Ara K, Ozaki K, Kakeshita H, Yamane K, Sekiguchi J (2007) Bacillus subtilis AprX involved in degradation of a heterologous protein during the late stationary growth phase. J Biosci Bioeng 104(2):135-143

Liu J, Fu K, Wu C, Qin K, Li F, Zhou L (2018) "In-Group" communication in marine vibrio: a review of $\mathrm{N}$-acyl homoserine lactones-driven quorum sensing. Front Cell Infect Microbiol 8:139

López M, Mayer C, Fernández-García L, Blasco L, Muras A, Ruiz FM, Bou G, Otero A, Tomás M, GEIH-GEMARA (2017) Quorum sensing network in clinical strains of $A$. baumannii: AidA is a new quorum quenching enzyme. PloS ONE 12(3):e0174454

Mei GY, Yan XX, Turak A, Luo ZQ, Zhang LQ (2010) AidH, an alpha/betahydrolase fold family member from an Ochrobactrum sp. strain, is a novel $\mathrm{N}$-acylhomoserine lactonase. Appl Environ Microbiol 76(15):4933-4942

Molière N, Turgay K (2009) Chaperone-protease systems in regulation and protein quality control in Bacillus subtilis. Res Microbiol 160(9):637-644

Nguyen HD, Phan TTP, Schumann W (2011) Analysis and application of Bacillus subtilis sortases to anchor recombinant proteins on the cell wall. AMB Express 1(1):22

Pan X, Yang Y, Liu X, Li D, Li J, Guo X, Zhou Z (2016) Secretory expression of a heterologous protein, Aiio-AlO6BS, in Bacillus subtilis via a non-classical secretion pathway. Biochem Biophys Res Commun 478(2):881-886

Park S, Schumann W (2015) Optimization of the secretion pathway for heterologous proteins in Bacillus subtilis. Biotechnol Bioprocess Eng 20(4):623-633

Tan CH, Koh KS, Xie C, Tay M, Zhou Y, Williams R, Ng WJ, Rice SA, Kjelleberg $S$ (2014) The role of quorum sensing signalling in EPS production and the assembly of a sludge community into aerobic granules. ISME J 8(6):1186-1197

Traag BA, Pugliese A, Setlow B, Setlow P, Losick R (2013) A conserved ClpP-like protease involved in spore outgrowth in Bacillus subtilis. Mol Microbiol 90(1):160-166

Wang LH, Weng LX, Dong YH, Zhang LH (2004) Specificity and enzyme kinetics of the quorum-quenching $\mathrm{N}$-acyl homoserine lactone lactonase (AHLlactonase). J Biol Chem 279(14):13645-13651

Wang WZ, Morohoshi T, Ikenoya M, Someya N, Ikeda T (2010) AiiM, a novel class of $\mathrm{N}$-acylhomoserine lactonase from the leaf-associated bacterium Microbacterium testaceum. Appl Environ Microbiol 76(8):2524-2530

Westers H, Darmon E, Zanen G, Veening JW, Kuipers OP, Bron S, Quax WJ, Van Dijl JM (2004a) The Bacillus secretion stress response is an indicator for a-amylase production levels. Lett Appl Microbiol 39(1):65-73

Westers L, Westers H, Quax WJ (2004b) Bacillus subtilis as cell factory for pharmaceutical proteins: a biotechnological approach to optimize the host organism. Biochim Biophys Acta Mol Cell Res 1694(1-3):299-310

Westers L, Westers H, Zanen G, Antelmann H, Hecker M, Noone D, Devine KM, van Dijl JM, Quax WJ (2008) Genetic or chemical protease inhibition causes significant changes in the Bacillus subtilis exoproteome. Proteomics 8(13):2704-2713

Wu XC, Ng SC, Near RI, Wong SL (1993) Efficient production of a functional single-chain antidigoxin antibody via an engineered Bacillus subtilis expression-secretion system. Nat Biotechnol 11(1):71-76

Wu SC, Yeung JC, Duan Y, Ye R, Szarka SJ, Habibi HR, Wong SL (2002) Functional production and characterization of a fibrin-specific single-chain antibody fragment from Bacillus subtilis: effects of molecular chaperones and a wall-bound protease on antibody fragment production. Appl Environ Microbiol 68(7):3261-3269

Yates EA, Philipp B, Buckley C, Atkinson S, Chhabra SR, Sockett RE, Goldner M, Dessaux Y, Cámara M, Smith H (2002) N-acylhomoserine lactones undergo lactonolysis in a $\mathrm{pH}$-, temperature-, and acyl chain lengthdependent manner during growth of Yersinia pseudotuberculosis and Pseudomonas aeruginosa. Infect Immun 70(10):5635-5646

Zhang M, Cao Y, Yao B, Bai D, Zhou Z (2011) Characteristics of quenching enzyme AiiO-AlO6 and its effect on Aeromonas hydrophila virulence factors expression. J Fish China 35(11):1720-1728

Zhang K, Su L, Wu J (2018) Enhanced extracellular pullulanase production in Bacillus subtilis using protease-deficient strains and optimal feeding. Appl Microbiol Biotechnol 102(12):5089-5103

\section{Publisher's Note}

Springer Nature remains neutral with regard to jurisdictional claims in published maps and institutional affiliations. 